\title{
Cartesian Controlled Heavy Machines Supported by Advanced Human-Machine-Interfaces
}

\author{
B. Fink, M. Hiller, H. Krölls, W. Risse and S. Schweikart \\ Institut für Mechatronik, IMECH GmbH \\ Eurotec Ring 15, 47445 Moers, Germany \\ e-mail: w.risse@imech.com
}

\begin{abstract}
This paper presents an approach to Cartesian controlled heavy machines. The system under investigation is a drill rig equipped with two drilling booms each actuated by eight hydraulic drives. To improve accuracy and efficiency in both possible operation modes, drilling and bolting, a Cartesian control system has been implemented, which takes advantage of using different subsets of the redundant kinematic boom structure. Another important aspect is the human-machineinterface which enables easy operation in manual and automatic control modes. In this context the use of Internet technologies is an important approach to build up standardized interfaces. First experiments with future concepts of human-machine-interfaces like gesture controlled operation yield promising results.
\end{abstract}

\section{Introduction}

Automation of heavy machines is closely related to the use of advanced human-machine-interfaces (HMI). Particularly in the case of Cartesian controlled manipulators new interaction techniques are required, because motion is no longer controlled by directly manipulating the valves of single actuators via separate levers. Therefore, a HMI has to be designed, which gives sufficient information about possible motions of the end-effector in Cartesian space and about the location of the tool-center-point. On the other hand, operation of the machine has to be quite intuitive, because complex interaction schemes will not be accepted by the workers on the site, who are in most cases not specially trained to handle automated machines.

This paper presents a Cartesian control system including an advanced HMI for a twin boom drill rig used in tunnel construction. Each boom is equipped with eight hydraulic actuators, which are feedback con- trolled to enable Cartesian motion of the complete system. Special redundant kinematics for each boom allow the combination of blasting and bolting operations. The control system enables the operator to move the drill rig intuitively in Cartesian space without considering appropriate positioning of single actuators. The aim of this concept is to give all possible support to the operator, which results in a significant speed-up of task duration. However, the complete replacement by a fully automated process is too complex in such hazardous environments and hence not economic. Nevertheless, this technique can even be used in the automatic drill operation mode.

Depending on whether drill and blast or bolting operation is activated different non-redundant subsets of the eight degree of freedom (DOF) kinematics are involved in performing Cartesian motion. Since most of the DOF are indirectly driven by non-linear transmission mechanisms, direct and inverse kinematics of the manipulator both can be solved by a two step process, which is described in section 3 . In addition to Cartesian control in either world or tool coordinates, a collision monitoring system prevents the two drilling booms from both colliding with each other or with the sweeping drilling tool itself.

With the objective to make the benefits of the Cartesian control system more approachable to the system operator a new HMI has been designed, which can be used intuitively (see section 4). The interface hardware consists of a LCD colour display, two passive joysticks (3 DOF each) and an electronic hand-wheel serving as a robust and simple input device. While manipulating the boom, e.g. reorienting the drill, the operator usually cannot cope with such abstract information like roll, pitch and yaw angles. Therefore, the HMI assists the operator by means of 3-D computer graphic models, which can be optionally animated. 
To give an outlook to the near future of HMI with respect to mining and tunnelling industry a modern concept for a hardware independent distributed user interface is presented. The Internet technology based system uses a WWW-server embedded on the control system of the drill rig to serve appropriate interfaces for different tasks, e.g. operation or maintenance, via a local area network (Intranet). The operator can control or supervise the machine by using a common Internet browser on his client computer without installing software depending on a special type of machine.

The last topic of section 4 deals with the application of multimodal user interfaces to heavy machines. Here a gesture recognition system has been implemented and successfully tested to control the movements of the drill rig by show of hands.

\section{System Description}

\subsection{Hardware Configuration}

In tunnel construction drill rigs with one up to three drilling booms are used for boring blastholes or fixing bolts. The drilling boom under investigation is part of a two arm drill rig used in road tunnel excavation (Interroc Germany). Fig. 1 shows the test bed for a full scale boom built up at the IMECH laboratory. The boom is mounted at a console which can

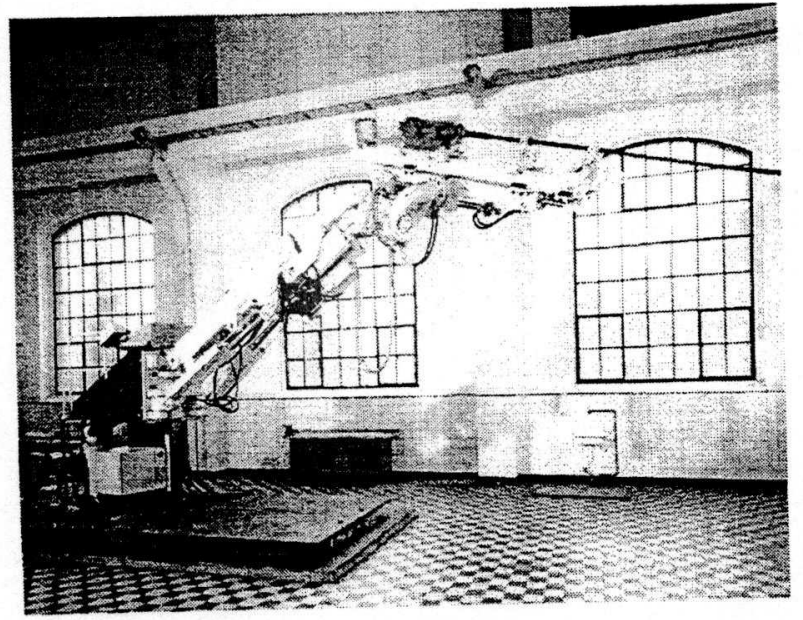

\section{Figure 1: Redesigned drilling boom of a drill rig}

be rotated by a hydraulic cylinder via a bidirectional valve in order to simulate possible slope positions of the vehicle. The deviation from the horizontal plane is measured by means of an inclinometer and fed back into the control system where a correction of the Cartesian motion is calculated. To obtain the current piston positions each of the seven cylinders has been equipped with a linear magnetostrictive position sensor system.

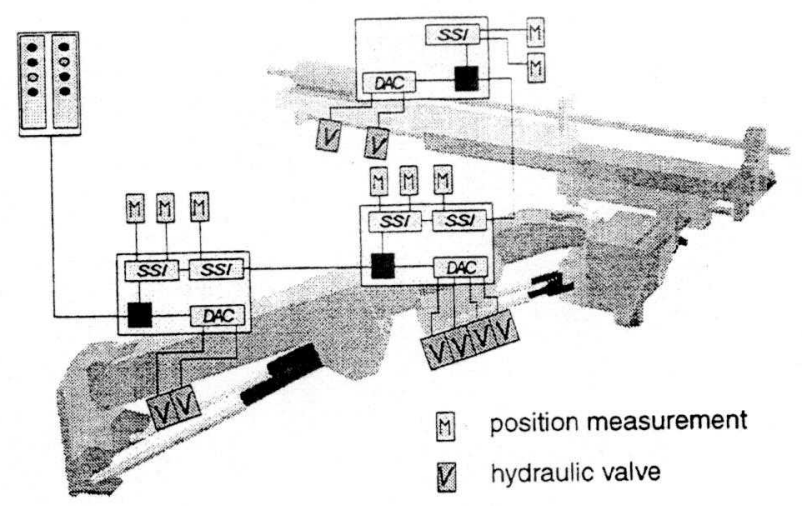

Figure 2: Configuration of sensors and actuators

In addition a multi-turn sensor has been added to the hydraulic motor. A sensor/actuator bus has been applied to reduce cabling costs and to increase reliability of the system. Another benefit of this approach is the easy integration of additional sensor and actuator signals into the control system. The long hydraulic tubes and central switching valves have been substituted by high precision feedback valves decentrally located on three blocks distributed over the boom.

The complete hardware configuration is shown in Fig. 2. Using the control scheme described in [1] a repeatable static position error of the drill lower than $5 \mathrm{~mm}$ is achievable.

\subsection{Control Structure}

Coordinated motion of the drilling boom requires a powerful control system with functionality ranging from human machine interface to actuator control. The hierarchical structure of the boom controller has been developed in simulation on a workstation within an Integrated Development Environment and was easily transferred to the controller by simply cross compiling the unchanged source code for the real-time target [2]. The control program consists of several processes running at different rates and priorities [3]. Fig. 3 shows the modules and the events activating them with priority and rate increasing from top to bottom. The upper module within the real-time environment is represented by a CommunicationServer that manages data exchange with the Human Machine Interface and processes user requests. Motion commands are handled by the PathPlanner providing an analytical description of the corresponding trajectory, which is subsequently evaluated by the PathGenerator at a certain rate in either joint or Cartesian coordinates. In the latter case the InverseKinematics module calculates an appropriate set of joint angles respectively piston positions for the given Cartesian coordinates. These com- 


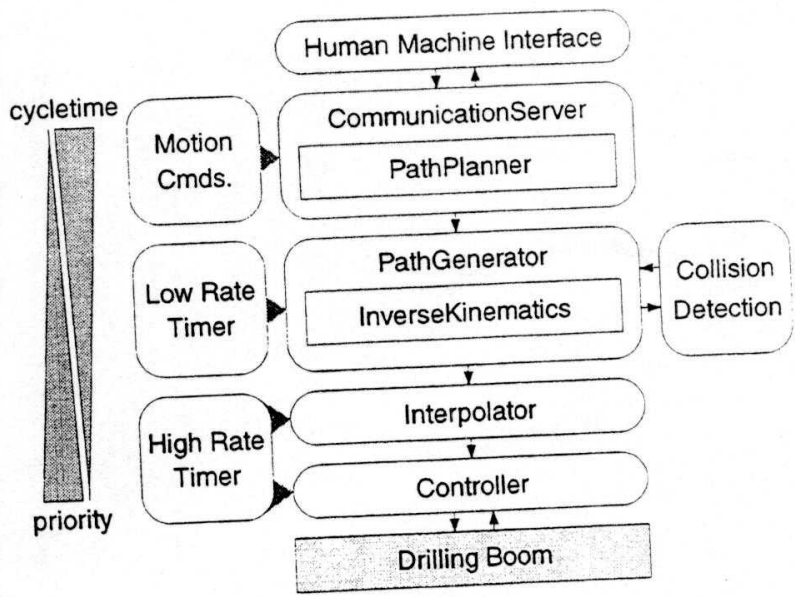

Figure 3: Motion control structure of the drill rig

putations are usually the most time-consuming part, thus the PathGenerator runs at a lower rate than the Controller. This multi-rate concept requires the introduction of an additional Interpolator to achieve smooth quantities. In the current implementation the Controller and the Interpolator are called every 5 ms while the PathGenerator is evaluated every four control cycles. This ratio may be increased in case further tasks are introduced.

Since two booms are mounted on one platform and operated simultaneously the rig has to be prevented from collisions either between two booms or between a boom and the adjacent tunnel wall. In addition collisions of boom and drill carrier of the same arm might take place due the bulky geometric structure. In order to ensure safe operation under these conditions a Collision Detection process has been introduced that may run on a separate CPU at a rate depending on the complexity of the work space and thus can be decoupled from the main control structure.

\section{Kinematics}

The kinematic structure of the drilling boom provides eight degrees of freedom (DOF) made up by six revolute and two prismatic joints (see Fig. 4a). Both, efficient and precise manual operation as well as automatic processing of programmed patterns require simultaneous and synchronous motion of these joints by means of Cartesian control.

\subsection{Operation modes}

For the given six-dimensional Cartesian task space the eight manipulator joints contain two redundant DOF. However, not all of the eight DOF are constantly in use. Depending on whether drill and blast or bolting operation is activated, different non-redundant subsets of these DOF are involved in performing the Cartesian motion. In the drilling mode the five DOF nearest to the platform are steadily used for positioning of the tool center point (TCP) - here located at the drill tip - and for alignment of the TCP frame with respect to the tunnel cross section. In this mode the rolling motion of the sixth axis is only casually activated to change between several sectors of the working plane in order to extend the manoeuvrability when operating in the vicinity of tunnel walls. To switch between drilling and bolting mode the seventh axis can be used to align the tool in either horizontal or vertical orientation. In the latter case the TCP is located at the intersection of the sixth and seventh axis and tool positioning is mainly performed by the roll axis with only little support from the first five DOF. The outermost translational axis is merely involved in the drilling or bolting process itself during which the other DOF are fixed. In both modes Cartesian motion may be prescribed in either fixed base coordinates or local tool coordinates.

Since five of the six revolute joints are indirectly driven by hydraulic cylinders additional transmission linkages have to be introduced. Hence, Cartesian control of the drilling boom must consider the open kinematic chain represented by eight DOF and four closed loops $L_{i}$ of the non-linear transmission linkages (see Fig. 4b). Due to this coupling, solving the direct and inverse kinematics of the manipulator can both be represented by a two step process. The calculations embrace the transformation between the tool position and orientation $\mathrm{T}_{T C P}$ in Cartesian space and the rotation angles $\boldsymbol{\theta}$ of the universal joints, as well as the relation between these quantities and the corresponding actuator variables $\boldsymbol{\beta}$, i.e. the piston positions

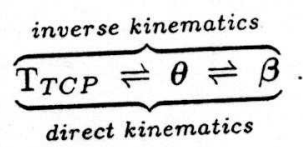

If the carrier vehicle is in a slope position, $\mathrm{T}_{T C P}$ may be premultiplied by a correction matrix that considering the deviation from the horizontal plane which is maesured by two inclinometers.

\subsection{Open Kinematic Chain}

The left part of eq. (1) can be described by means of general transformations between adjacent joints in terms of homogeneous transformation matrices

$$
\mathrm{A}=\left[\begin{array}{cc}
R & \underline{r} \\
\underline{0}^{T} & 1
\end{array}\right],
$$

where $R$ is a $3 \times 3$ rotation matrix and $\underline{r}$ the translation vector. Using this notation the direct kinemat- 
a)

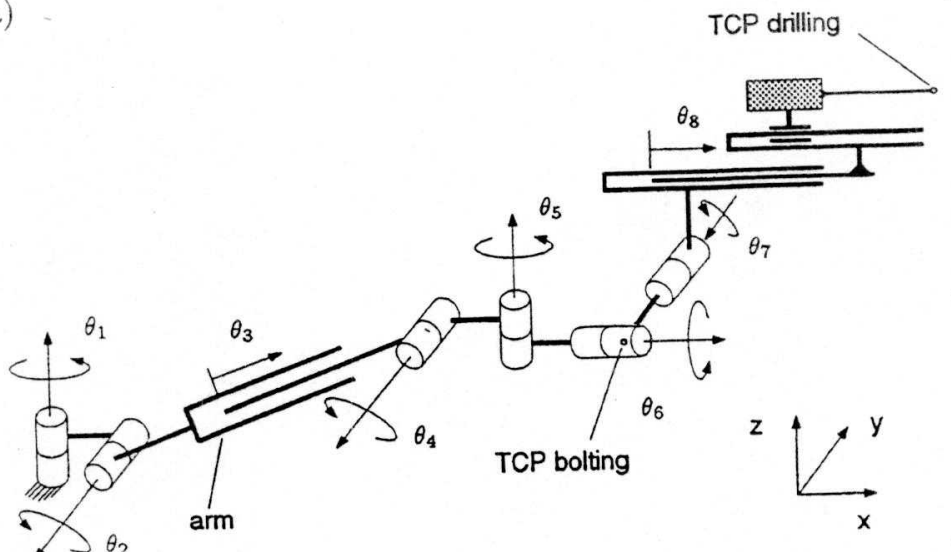

b)

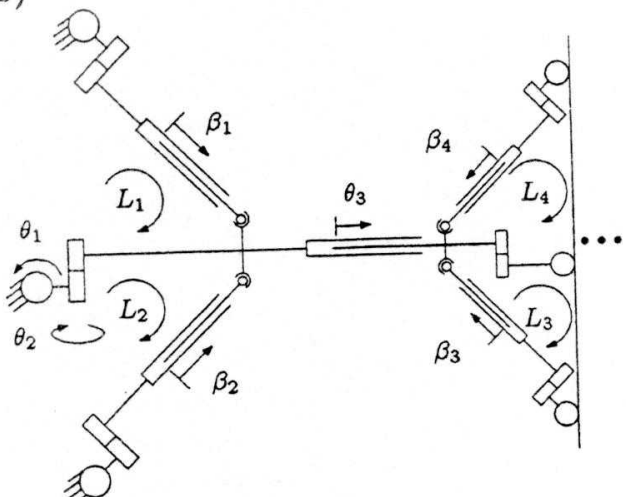

Figure 4: Kinematic Structure of the drilling boom (a) and the closed loops (b)

ics equations for the drilling mode can be obtained by subsequent multiplication of all relative joint transformations

$$
T_{T C P}=A_{1} A_{2} A_{3} A_{4} A_{5} A_{6} A_{7} A_{8} .
$$

Due to the fact that the translational drilling axis is used only during the actual drilling process and joint seven serves merely as a mode switch, these DOF can be considered as constant during the arm motion. Thus inverse kinematics in both drilling and bolting mode has to solve for the six DOF nearest to the platform which can be obtained in closed form by successive premultiplication of eq. (3) by the inverse joint transformations [4]. This procedure leads to a structure

$$
\begin{aligned}
A_{1}^{-1} T_{6} & =A_{2} A_{3} A_{4} A_{5} A_{6} \\
A_{2}^{-1} A_{1}^{-1} T_{6} & =A_{3} A_{4} A_{5} A_{6} \\
& \vdots \\
A_{5}^{-1} A_{4}^{-1} A_{3}^{-1} A_{2}^{-1} A_{1}^{-1} T_{6} & =A_{6},
\end{aligned}
$$

where in each stage one additional joint variable can be solved by choosing an appropriate scalar equation from the corresponding transformation. Since $\theta_{2}$ and $\theta_{4}$ are rotations with respect to parallel axes, the solution is slightly different from the above scheme. In this special case we first determine the sum $\theta_{24}$ and obtain the single angles after solving for the other rotational variables.

\subsection{Closed Loop Solution}

For the right part of eq. (1), i.e. the relation between the joint angles and the piston positions, a closed form solution exists only for the inverse kinematics branch whereas direct kinematics must be solved by numerical iteration. This is due to the closed loops of the transmission linkages and states a problem common to most parallel mechanisms. To guarantee the convergence of the iterative algorithm it is essential to choose the starting point close enough to the exact solution. For this purpose an estimation is computed from a pre-calculated characteristic curve.

Since there is no tool processing required while moving the TCP, inverse kinematics may be solved on position level only. However, to increase path accuracy velocity inputs $\dot{\boldsymbol{\beta}}$ for the joint controllers are optionally generated by differentiation.

\subsection{Collision Detection}

In addition to Cartesian control a collision threat detection system prevents the two drilling booms from either colliding with each other, the tunnel wall or the sweeping basket. In order to ensure safe manipulator operation the distances between discrete collision endangered points and possible obstacles are calculated in real-time based on a reduced model of the mechanisms and the workspace. In case one of these distances falls below a certain bound the operator will be notified via the HMI. If continued motion towards the obstacle reduces this distance further below a safety limit, the entire motion of this arm will be stopped immediately. In this state operation can only be reactivated after an explicit failure acknowledgement.

\section{User Interface for the Drill Rig}

Today computerized heavy duty machines such as the drill rig described in this paper are still the exception at construction sites. Hence, there is only little experience in the design of user interfaces for this type of machinery. To achieve high productivity with this equipment it is important to provide optimal conditions for the interaction between a human operator and the control system of the machine. The following section deals with the current state of the user interface 
as it is installed on the test-bed drilling boom. The challenge is to design an user interface that is easy to handle by offering an intuitive method for programming automatic functions. The - often not trained operator has to be guided through menus and options of the interface without loosing orientation in the menu structure. Furthermore, the interface is onboard the machine, so its design must fit the harsh conditions found in mines or tunnel construction sites. For that reason only very robust input devices have been chosen. In Fig. 5 the panel is depicted as it is installed

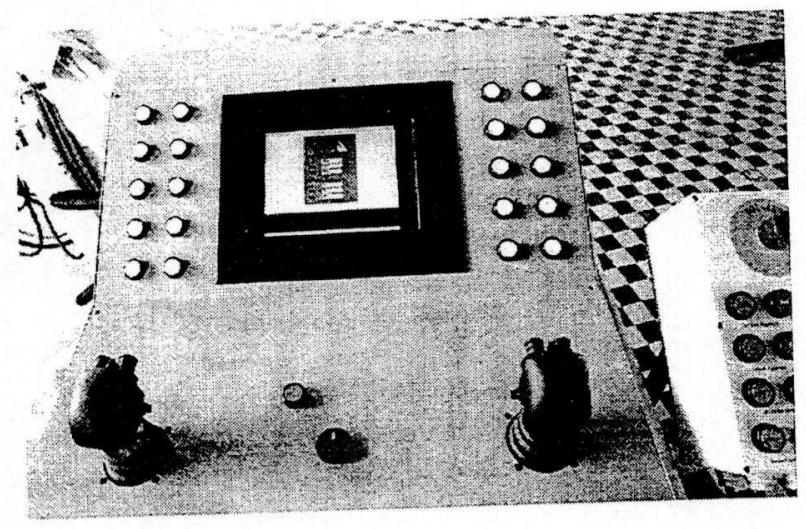

\section{Figure 5: Panel of the Human-Machine-Interface}

at the drilling boom in the test bed. Push-buttons for standard actions (e.g. boring mill on/off) and automatic functions (e.g. bore-hole depth supervision) are situated next to the flat panel monitor. The joysticks (three DOF each) are used for positioning and orientating the boom in Cartesian or joint space respectively. The HMI presents a number of form-fillin dialoges to the operator, which can be used e.g. to configure or observe the bore-hole depth during the drilling process. The user can feed data in the computer system using an electronic hand wheel that is situated between the joysticks. This input device completely replaces the conventional pointing device (e.g. mouse) and the keyboard. A more detailed description of the interface and the considerations made for its design can be found in [1].

\subsection{Concept for Distributed User Interfaces}

For handling complex heavy machinery an operator has different demands on the user interface depending on his acual task, e.g. moving, maintenance or teleoperation [5]. The approach presented here describes the concept of a distributed system of user interfaces. The implementation of the proposed system is based on commonly known client/server-architecture and software techniques found in standard internet/intranet technology. Independent from the type of machinery all control panels used for similar tasks like operating the machine or maintenance are equipped with a similar set of interactive control elements (e.g. joysticks, monitor, buttons), so that a benefit can be obtained by standardization. A worker from the maintenance group e.g. should be capable of performing diagnostic tasks via a common hand-held computer, independent from the type of heavy duty machinery he is working with. The specific features of the machine are displayed by appropriate graphical user interfaces which can be downloaded by a standard web-browser from the control system where all interface data is stored on a central web-server. This results in an intense cost reduction for software design and implementation of user interfaces. In Fig. 6 a possible setup for a distributed environment is presented. It is obvious that the application of telemanipulation methods or the integration of the drill rig into a PLC-system can be accomplished easily. The presented concept was experimentally applied to an industrial robot system. A web-server has been added to the control software system. The so called common gateway interface of the web- server has been extended with a set of functions to provide communication between server and robot control software. The user interfaces are programmed in the network language Java. Interfaces can be downloaded by the operator's web-browser implemented on a PC running Microsoft Windows 95 but no specific robot control software. The interface displays status information of the system and offers access to the robot motion controller.

\subsection{Gesture Control - Experiments with a Multi- modal User Interface}

A very sophisticated approach to a modern design of user interfaces is the application of a multimodal user interface. Multimodal interfaces employ combinations of natural interaction forms, such as speech, gesture or handwriting. The human user is enabled to communicate with a technical system in such a way that he can employ his natural communication habits. A crucial point in this form of communication is that the interface designer has to find the appropriate multimodal metaphors that have a definite meaning and correspond directly between human behaviour and the technical process. A gesture recognition system developed by the Computer Science Department of the University of Duisburg has been attached to the control system of the drill rig. The system performs user and background independent motion pattern recognition of body movements [6]. All movements of the operator are tracked by a video camera and computed by a neural network. The recognized patterns are converted to a control command for the drilling boom. 


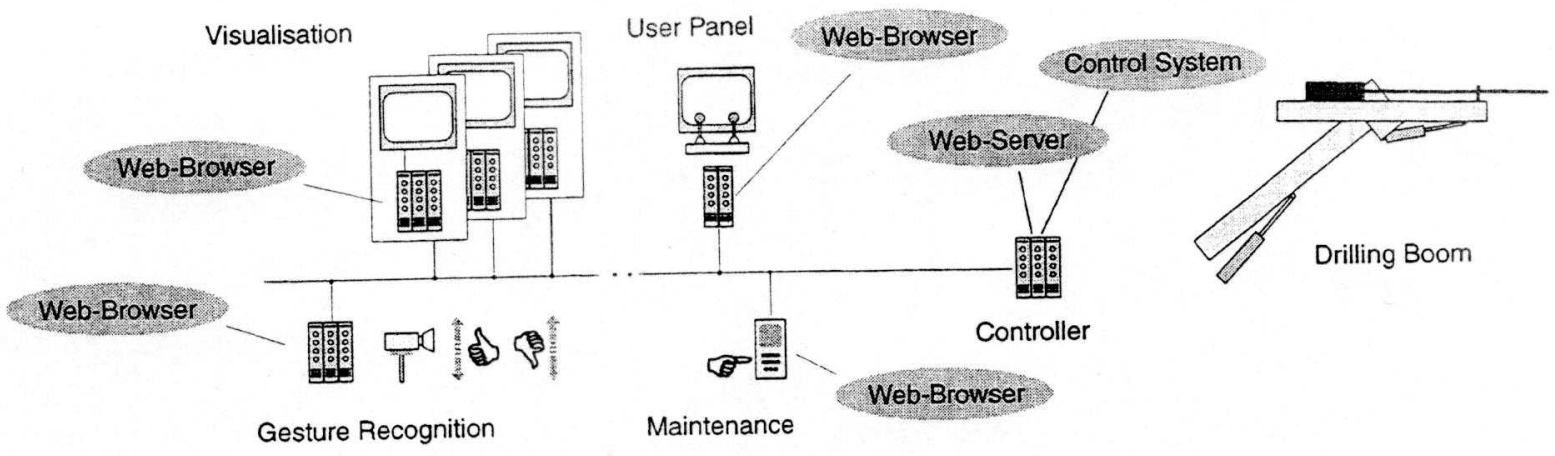

Figure 6: Distributed User Interface of the Drilling Boom

In the test setup of this multimodal user interface seven different dynamic movements can be recognized (boom movement: arm -left, -right, -up, -down, -back, -forth; boom stop: shake head). In the test bed machine the operator is standing in front of the drill rig and conducts the drilling process using significant body gestures (see Fig. 7). The key advantage of gesture

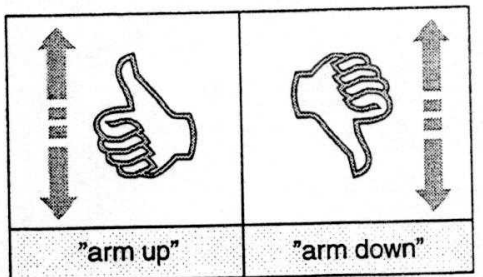

Figure 7: Gestures for typical boom motions

control is that the operator is situated directly at the place where action takes place, whereas with the conventional human-machine-interface onboard the drill rig an operator is approximately seven meters away from the actual working place. During the experiments the operators really felt to be in control of the process because the gestures/metaphors chosen for guiding the drilling boom have been chosen appropriate to the action desired.

\section{Conclusions}

Multimodal interfaces represent the ultimate form of an ideal human machine interaction. Although the technical problems with such systems are enormous in respect to the reliability of pattern recognition algorithms and robustness according to the environment, the results of the experiments carried out with the gesture recognition system described above are very promising. Thus future work will focus on tele assistance where gesture recognition will be extended from motion prescription to the control of complete tasks, e.g. drilling of hole patterns. Another essential aspect will be the massive use of information like video or VR feed-back to support the operator performing tele manipulation under working conditions where the TCP is not in sight.

\section{Acknowledgement}

This project is realized in cooperation with Deilmann-Haniel, Dortmund, Germany. It is financially supported by the Ministry of Economics of the Federal State North Rhine-Westphalia.

\section{References}

[1] B. Fink, M. Hiller, H. Krölls and S. Schweikart. Concepts of an advanced cartesian control system for a redundant twin boom drill rig. In Proc. of the 13th Int. Symp. on Automation and Robotics in Construction (ISARC), Tokyo, Japan, pages 103-112, 1996.

[2] M. Anantharaman, B. Fink, M. Hiller, and S. Vogel. Integrated Development Environment for Mechatronic Systems. In Proceedings of the 3rd Conference on Mechatronics and Robotics, pages 54-69, Paderborn, Germany, 1995.

[3] W. Risse and M. Hiller. Control of a redundant scara robot in the presence of obstacles. In 11th CISM-IFToMM Symposium on Theory and Practice of Robots and Manipulators (RoManSy), Udine, Italy, pages 277-284, 1996.

[4] Richard P. Paul. Robot Manipulators: Mathematics, Programming, and Control. The MIT Press Series in Artificial Intelligence. The MIT Press, Cambridge (Massachusetts), London (England), 1986.

[5] R. Roshardt and G. Schweitzer. Feasibility study for operating a sensor-guided heavy duty manipulator (in German). Technical report, ETH Zürich (IfR), Switzerland, 1995.

[6] G. Rigoll and A. Kosmala. New improved feature extraction methods for real-time high performance image sequence recognition, IEEE Int. Conf. on Acoustics, Speech and Signal Processing (ICASSP), Munich, Germany. 1997. 